\title{
General Quasiparticle Propagator and Mass Dependence in Degenerate Color Superconductivity
}

\author{
Todd Fugleberg \\ Department of Physics \\ Brookhaven National Laboratory, Upton, New York 11973, U.S.A. \\ email:fugle@quark.phy.bnl.gov \\ BNL-NT-02/12
}

\begin{abstract}
The general quasiparticle propagator in dense quark matter is derived for equal mass quarks. Specialized to an NJL model, this propagator includes one new condensate, $\Delta_{3}$, in addition to the usual CFL condensate, $\Delta_{1}$. The gap equation is solved in two NJL models and the dependence on the quark mass of the condensates and the gap is presented. Analytic approximations for the condensates are obtained and compared to exact numerical solutions. The results are shown to differ from those obtained by neglecting $\Delta_{3}$, especially for smaller values of $\Delta_{1}$. The two different NJL models presented are also shown to give different results when $\Delta_{3}$ is not neglected. The methods used in this paper can be generalized to the physical case where only the strange quark is significantly massive.
\end{abstract}

\section{Introduction}

The physics of strongly interacting matter at high densities and low temperatures has been the subject of much research in recent years. It has long been known [1, 2] that at sufficiently high densities a system of quarks should form a condensate of Cooper pairs which breaks the $S U_{C}(3)$ symmetry and becomes a color superconductor. The formation of the diquark condensates leads to gaps in the quasi-particle spectrum. The authors of [1, 2] estimated that the gaps were of the order of $\Delta \sim 10^{-3} \mu$ where $\mu$ is the quark chemical potential. More recently it was shown at realistic values of $\mu$ in an instanton induced 
NJL' model that gaps of the order of 10-100 MeV could be obtained[3]. This stimulated a great deal of research ${ }^{2}$ in the ensuing years and has resulted in a proliferation of predicted superconducting ground states. These states may be realized in the cores of neutron or quark stars and lead to observable effects[6, 7, 8, 9].

It is widely accepted 10, 11] that for three colors and flavors the color superconducting ground state at asymptotic densities is the Color Flavor Locked (CFL) state 10]:

$$
\begin{aligned}
\left\langle q_{\alpha}^{i} C \gamma^{5} q_{\beta}^{j}\right\rangle & =\Delta_{\overline{3} \overline{3}}\left(\delta_{\alpha}^{i} \delta_{\beta}^{j}-\delta_{\beta}^{i} \delta_{\alpha}^{j}\right)+\Delta_{66}\left(\delta_{\alpha}^{i} \delta_{\beta}^{j}+\delta_{\beta}^{i} \delta_{\alpha}^{j}\right) \\
& \approx \Delta_{\overline{3} \overline{3}}\left(\sum_{A=2,5,7} \lambda_{\alpha \beta}^{A} \lambda_{i j}^{A}\right)
\end{aligned}
$$

where the Greek indices are color indices, the Latin indices are flavor indices, the $\overline{3}$ and 6 subscripts refer to anti-triplet or sextet configurations in color and flavor spaces respectively and $\lambda^{A}$ are the Gell-Mann matrices. At lower densities $\left(\mu \sim m_{s}^{2} / 4 \Delta\right)$, it is likely that the ground state is a superconducting state involving the condensation of Cooper pairs in the $u-d$ sector only (2SC):

$$
\left\langle q_{\alpha}^{i} C \gamma^{5} q_{\beta}^{j}\right\rangle=\Delta_{2 S C} \epsilon_{\alpha \beta 3} \epsilon^{i j 3},
$$

Finally at still lower densities the favored ground state will be ordinary hadronic matter. Two new phases have recently been predicted: Crystalline Color Superconductivity 12 and CFL with meson condensation 13, 14. These predictions, while not necessarily at odds with one another, indicate that the transition region between the CFL state and hadronic matter is not completely understood.

A standard way of studying color superconductivity is to solve the gap equation in the Nambu Gorkov formalism which is reviewed in Appendix A. The inclusion of a strange quark mass in the gap equation introduces two sets of complications: 1) massive quarks means that there are 4 new allowed Dirac structures for the condensates, and coupling between the condensates; 2 ) the fact that the strange quark is different from the other quarks means that condensates involving the strange quark should be different from those with zero strangeness.

\footnotetext{
${ }^{1}$ The designation NJL (Nambu-Jona-Lasinio) model is used in this paper to describe models where the quark interaction is taken to be a four-fermion interaction at a point.

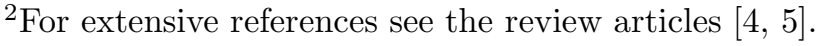


In order to understand the implications of these two complications it is useful to separate them and understand them individually before tackling the full problem. In a previous paper by this author 15] the second problem of non-degenerate quarks was studied in the case where all quarks are massless but the strange quark is given a different chemical potential than the other two quarks. This paper concentrates on the first complication by considering the problem of equal mass quarks.

Color superconductivity with massive quarks has been studied before. The papers [16]-23 have dealt with inclusion of quark mass or strange quark mass in NJL models. The papers [13, 14, 24] have dealt with inclusion of quark mass or strange quark mass in effective Lagrangian models. Finally [25, 26] have dealt with this problem in the perturbative approach. All of these important papers have dealt with the problem at some level of approximation. The new results in this paper are solutions for all allowed diquark condensates applicable over a wide range of quark masses and coupling constants. The quasiparticle propagator derived herein is the general form for the case of equal mass quarks. It is shown that for the NJL model there is one and only one additional diquark condensate, $\Delta_{3}$, and it is included in the calculations. The approximate analytic solutions presented in this paper make numerical solution of gap equations for different parameter values unnecessary. Finally two different NJL models are analyzed in exactly the same framework so that they can be compared and contrasted.

The authors of [16] did discuss the fact that $\Delta_{3}$, which they refer to as $\kappa$, must be non-zero but since it is small they neglect it in their calculations. The authors of [21] include a similar diquark condensate in the 2SC phase, but their analysis does not indicate that no other diquark condensates would arise. In [21] it was shown that $\Delta_{3}$ can be large in an NJL model using a Lorentz non-invariant interaction and therefore have a significant effect on the gap. In this paper it is shown that, even for small values of $\Delta_{3}$, neglecting this condensate in the calculation can have a non-trivial effect on the gap.

The results of this paper are an approximation to the $N_{f}=3 \mathrm{CFL}$ case as the $\Delta_{66}$ gaps are neglected to simplify the presentation. The results are exact for $N_{f}=2$ and for the $2 \mathrm{SC}$ phase with $N_{f}=3$. Therefore there is only one color-flavor structure and we drop the subscripts " $3 \overline{3}$ " and "2SC" as they are unnecessary.

The general quasiparticle propagator is presented for the case of equal mass quarks. This propagator is then specialized to the NJL model where it is shown that there is only one new diquark condensate, $\Delta_{3}$, in addition to 
the CFL condensate, $\Delta_{1}$.

The gap equation was solved in two different NJL models. The first model used is the simplest possible NJL model equivalent to scalar exchange. The second model used is an NJL model with the color structure of single gluon exchange. The second model is perhaps more physically motivated and is the model usually used in NJL analyses of color superconductivity. However, there is no reason to exclude scalar interaction terms and restrict the analysis to interactions motivated by single gluon exchange as a general low energy Lagrangian could include both. This being said it is interesting to see how much of an effect the specific NJL model has on the results.

The gap equation was solved in the scalar NJL model and results are presented for the general set of condensates as a function of the quark mass. Approximate analytic solutions for the condensates are obtained and shown to agree well with exact numerical solutions. The results are compared with results obtained neglecting the effect of the new condensate $\Delta_{3}$. It is shown that the inclusion of $\Delta_{3}$ alters the results especially for lower values of $\Delta_{1}$. The gap in the quasiparticle spectrum is shown to be a function of both $\Delta_{1}$ and $\Delta_{3}$ and inclusion of $\Delta_{3}$ is even more relevant.

The same analysis was performed in an NJL model with the color flavor structure of single gluon exchange. The results in this model are also altered by the inclusion of $\Delta_{3}$ especially for lower values of $\Delta_{1}$ but they have the opposite effect as in the scalar NJL model.

These results are relevant to the analysis of [22] where the gap equation is solved neglecting $\Delta_{3}$. Their results are shown to be a reasonable approximation for the parameters they chose, but can be generalized using the techniques of this paper. The effects of chiral symmetry breaking have not been taken into account in this paper as in [22, 21] but such an extension would not be difficult.

These results illustrate the effect of using the general form of the quasiparticle propagator in the solution of the gap equations for massive but degenerate quarks. The results are most significant for smaller values of the CFL condensate and they depend strongly on the specific NJL model used.

The methods used in this paper are a significant new result on their own. They can be generalized to determine the general quasiparticle propagator and the general set of gap equations for the physical case where the up and down quarks are essentially massless, but the strange quark is massive. As well, this analysis could be repeated using perturbation theory in order to compare with a model that is exact at higher densities and energies. 
The outline of this paper is as follows. In section II a basis for the Dirac structure of the condensates is presented which facilitates the rest of the analysis. In section III the general quasiparticle propagator is derived and then specialized to the NJL model. In section IV the gap equations are solved in the scalar NJL model. In section V the gap equations are solved in an NJL model which has the color structure of single gluon exchange. Section VI concludes with a summary of the results. Appendix A contains a brief review of the Nambu-Gorkov formalism. Appendix B gives the decomposition from [2] of the gap matrix in terms of the products of Dirac matrices.

\section{Basis for the Condensates}

The CFL condensate in (1) has the Dirac structure $\gamma_{5}$ and is the only spin zero condensate for the massless case in an NJL model. The most general set of spin zero condensates is given in Appendix B and includes eight different possible structures. In an NJL model the structures involving $\gamma \cdot \hat{k}$ automatically vanish leaving four possible Dirac structures: $\gamma_{5}, \gamma_{5} \gamma_{0}, \gamma_{0}, I$. Solving the gap equations give the result that there is only one new condensate, $\Delta_{3}$ with the Dirac structure: $\gamma_{5} \gamma_{0}$.

The analysis could be carried out in a basis of Dirac structures, but the equations would be very complicated. A crucial step in finding the general quasiparticle propagator and gap equations is choosing a basis which simplifies the analysis enough to make it tractable.

In the massless case an arbitrary condensate matrix can be decomposed using a basis of orthogonal projection operators[27]:

$$
P_{h}^{e}(\vec{k})=\Lambda^{e}(\vec{k}) P_{h}(\vec{k}), \quad e, h= \pm 1,
$$

which are products of positive and negative energy projectors:

$$
\Lambda^{e}(\vec{k})=\frac{1+e \gamma_{0} \vec{\gamma} \cdot \vec{k}}{2} \quad e= \pm 1,
$$

and positive and negative helicity projectors:

$$
P_{h}(\vec{k})=\frac{1+e \gamma_{5} \gamma_{0} \vec{\gamma} \cdot \vec{k}}{2} \quad h= \pm 1 .
$$

In this basis the condensate matrix is:

$$
\Delta=\sum_{e, h, \pm 1} \Delta_{h}^{e} P_{h}^{e}
$$


Similarly all objects, such as the bare quark and the quasiparticle propagator can be written in this basis. The basis of orthogonal projection matrices is extremely useful since it reduces products of these objects to the simplest possible form.

In the case of massive quarks things are more complicated. The energy projector can be generalized to [27]:

$$
\Lambda^{e}(\vec{k})=\frac{1+e\left(\beta \gamma_{0} \vec{\gamma} \cdot \vec{k}+\alpha \gamma_{0}\right)}{2} \quad e= \pm 1
$$

where $\beta \equiv|\vec{k}| / E_{k}$ and $\alpha \equiv m / E_{k}$. A set of analogous operators in the massive case given in [27] is:

$$
P_{c h}^{e}(\vec{k})=P_{c} \Lambda^{e}(\vec{k}) P_{h}(\vec{k}), \quad e, h= \pm 1 \quad c=r, l,
$$

where:

$$
P_{c}=\left(\frac{1+c \gamma_{5}}{2}\right) \quad c=r, l,
$$

is the chirality projector which projects onto right and left handed spinors. The definition $r=+$ and $l=-$ in analogy with the other projectors will be used in what follows. These operators are really quasiprojectors with the general product rule:

$$
P_{c h}^{e}(k) P_{c^{\prime} h^{\prime}}^{e^{\prime}}(k)=\delta_{e e^{\prime}} \delta_{c c^{\prime}} \delta_{h h^{\prime}} P_{c h}^{e}(k)-\frac{1}{2} e c e^{\prime} c^{\prime}\left(1-e^{\prime} c h \beta\right) \delta_{h h^{\prime}} P_{c h}^{e^{\prime}}(k) .
$$

They are not projectors because chirality and energy projectors do not commute:

$$
\left[P_{c}, \Lambda^{e}\right]=-e c \alpha \gamma_{0} \gamma_{5} .
$$

This basis is complete and the general gap matrix can be written 27]:

$$
\Delta=\sum_{e, c, h, \pm 1} \Delta_{c h}^{e} P_{c h}^{e}
$$

This basis still involves many complications.

The calculation is simplified by defining a new basis of true projectors and nilpotent operators:

$$
P_{h}^{e}(k)=\sum_{c=-1,1} P_{c h}^{e}(k)=P_{h}(k) \Lambda^{e}(k),
$$




$$
Q_{h}^{e}(k)=\sum_{c=-1,1}(e c h-\beta) P_{c h}^{e}(k)=\left(e h \gamma^{5}-\beta\right) P_{h}^{e}(k),
$$

that satisfy the following relations:

$$
\begin{aligned}
P_{h}^{e}(k) P_{h^{\prime}}^{e^{\prime}}(k) & =\delta_{e e^{\prime}} \delta_{h h^{\prime}} P_{h}^{e}(k), \\
Q_{h}^{e}(k) P_{h^{\prime}}^{e^{\prime}}(k) & =\delta_{e e^{\prime}} \delta_{h h^{\prime}} Q_{h}^{e}(k), \\
P_{h}^{e}(k) Q_{h^{\prime}}^{e^{\prime}}(k) & =\delta_{-e e^{\prime}} \delta_{h h^{\prime}} Q_{h}^{-e}(k), \\
Q_{h}^{e}(k) Q_{h^{\prime}}^{e^{\prime}}(k) & =-\alpha^{2} \delta_{-e e^{\prime}} \delta_{h h^{\prime}} P_{h}^{-e} .
\end{aligned}
$$

The $P_{h}^{e}(k)$ are orthogonal projectors and the $Q_{h}^{e}(k)$ are nilpotent even though products of $P_{h}^{e}(k)$ and $Q_{h}^{e}(k)$ or $Q_{h}^{e}(k)$ and $Q_{h^{\prime}}^{e^{\prime}}$ mix the operators. $P_{h}^{e}(k)$ is an hermitian operator and $\left(Q_{h}^{e}(k)\right)^{\dagger}=-Q_{h}^{-e}(k)$. In the chiral limit $(\alpha \rightarrow 0$, $\beta \rightarrow 1) P_{h}^{e}(k)$ are identical to the massless projectors and $Q_{h}^{e}(k) \rightarrow 0$. Notice that the operators with different helicities are completely decoupled.

The multiplication table for each helicity is:

$$
\begin{aligned}
& P_{h}^{+} \quad Q_{h}^{+} \quad P_{h}^{-} \quad Q_{h}^{-} \\
& \begin{array}{l}
P_{h}^{+} \\
Q_{h}^{+} \\
P_{h}^{-} \\
Q_{h}^{-}
\end{array} \quad\left(\begin{array}{cccr}
P_{h}^{+} & 0 & 0 & Q_{h}^{-} \\
Q_{h}^{+} & 0 & 0 & -\alpha^{2} P_{h}^{-} \\
0 & Q_{+}^{+} & P_{h}^{-} & 0 \\
0 & -\alpha^{2} P_{h}^{+} & Q_{h}^{-} & 0
\end{array}\right) .
\end{aligned}
$$

This set of eight operators is the set of operators on the space of solutions of the Dirac equation which gives the most sparse multiplication table. One would prefer a basis of only orthogonal projection operators as in the massless case, but such a set does not exist. Dirac spinors are 4 component complex vectors and as such live in $\mathcal{R}^{8}$. One can definitely find eight orthogonal projectors in this space, but Dirac spinors must be solutions of the Dirac equation which places constraints on the components and reduces the dimensionality of the space. Therefore, you cannot have 8 orthogonal projectors onto the space of solutions of the Dirac equation. On the other hand you need 8 operators to form a complete set with which to construct all 8 independent Dirac structures. In the massless case, the left and right handed spinors decoupled so that each was a two component complex spinor living in $\mathcal{R}^{4}$ with two constraints from the Dirac equation. It is therefore possible to find a set of 4 orthogonal projectors on this space. 
The operators given above have the following representation as $2 \times 2$ matrices:

$$
\begin{gathered}
P_{h}^{+}=\left(\begin{array}{ll}
1 & 0 \\
0 & 0
\end{array}\right), \quad P_{h}^{-}=\left(\begin{array}{ll}
0 & 0 \\
0 & 1
\end{array}\right), \\
Q_{h}^{+}=\left(\begin{array}{ll}
0 & 0 \\
-\alpha & 0
\end{array}\right), \quad Q_{h}^{-}=\left(\begin{array}{ll}
0 & \alpha \\
0 & 0
\end{array}\right) .
\end{gathered}
$$

This supports the reasoning above that there are only 4 independent projectors since the operators for each handedness operate on a separate two dimensional space. This representation will be useful in what follows.

This set of operators is complete as shown by the following relations:

$$
\begin{aligned}
I & =\sum_{e, h=-1,1} P_{h}^{e}(k), \\
\gamma^{5} \gamma^{0} \gamma \cdot \hat{k} & =\sum_{e, h=-1,1} h P_{h}^{e}(k), \\
\alpha \gamma \cdot \hat{k} & =-\sum_{e, h=-1,1} Q_{h}^{e}(k), \\
\alpha \gamma^{5} \gamma^{0} & =\sum_{e, h=-1,1} h Q_{h}^{e}(k), \\
\gamma^{0} \gamma \cdot \hat{k} & =\sum_{e, h=-1,1} e\left(Q_{h}^{e}(k)+\beta P_{h}^{e}(k)\right), \\
\gamma^{5} & =\sum_{e, h=-1,1} e h\left(Q_{h}^{e}(k)+\beta P_{h}^{e}(k)\right), \\
\alpha \gamma^{0} & =\sum_{e, h=-1,1} e\left(\alpha^{2} P_{h}^{e}(k)-\beta Q_{h}^{e}(k)\right), \\
\alpha \gamma^{5} \gamma \cdot \hat{k} & =-\sum_{e, h=-1,1} e h\left(\alpha^{2} P_{h}^{e}(k)-\beta Q_{h}^{e}(k)\right) .
\end{aligned}
$$

It should be noted that these equations are still valid in the chiral limit (albeit half of them are trivial).

The gap matrix is decomposed in terms of these operators as:

$$
\begin{aligned}
\Delta & =\sum_{e, h=-1,1}\left(\beta \xi_{h}^{e}+\alpha^{2} \psi_{h}^{e}\right) P_{h}^{e}(k)+\left(\xi_{h}^{e}-\beta \psi_{h}^{e}\right) Q_{h}^{e}(k), \\
\Delta^{\dagger} & =\sum_{e, h=-1,1}\left(\beta \xi_{h}^{e}+\alpha^{2} \psi_{h}^{e}\right) P_{h}^{e}(k)-\left(\xi_{h}^{e}-\beta \psi_{h}^{e}\right) Q_{h}^{-e}(k) .
\end{aligned}
$$


All objects in this analysis can be represented using this basis. The sparse multiplication table simplifies much of the analysis in the rest of this paper. In the next section the general quasiparticle propagator is derived using this basis.

\section{General Quasiparticle Propagator}

The quasiparticle propagator is determined by the equation 27

$$
G^{ \pm} \equiv\left\{\left[G_{0}^{ \pm}\right]^{-1}-\Delta^{\mp} G_{0}^{\mp} \Delta^{ \pm}\right\}^{-1}
$$

where $\Delta^{+} \equiv \Delta, \Delta^{-} \equiv \gamma_{0} \Delta^{+} \gamma_{0}$ and $G_{0}^{-}$is the bare charge-conjugate particle propagator:

$$
\begin{aligned}
G_{0}^{-}(k) & =\left(\gamma^{\nu} k_{\nu}-\mu \gamma^{0}-M\right)^{-1}=\frac{\gamma^{\nu} k_{\nu}-\mu \gamma^{0}+M}{\left(k_{0}-\mu\right)^{2}-E_{k}^{2}} \\
& =\gamma^{0} \frac{\left(\left(k_{0}-\mu\right)-E_{k} \beta \gamma^{0} \gamma \cdot \hat{k}+E_{k} \alpha \gamma^{0}\right)}{\left(k_{0}-\mu\right)^{2}-E_{k}^{2}} \\
& =\gamma^{0} \frac{\left(k_{0}-\mu\right)+E_{k} \sum_{e, h=-1,1} e\left[\left(\alpha^{2}-\beta^{2}\right) P_{h}^{e}(k)-2 \beta Q_{h}^{e}(k)\right]}{\left(k_{0}-\mu\right)^{2}-E_{k}^{2}}
\end{aligned}
$$

Defining:

$$
\begin{aligned}
\Omega & =\left[G^{+}(k)\left(\left(k_{0}-\mu\right) \gamma^{0}-\gamma \cdot \vec{k}+M\right)^{-1}\right]^{-1} \\
& =\sum_{e, h=-1,1}\left(A_{h}^{e} P_{h}^{e}(k)+B_{h}^{e} Q_{h}^{e}(k)\right)=\sum_{h=-1,1} \Omega_{h},
\end{aligned}
$$

the coefficients are:

$$
\begin{aligned}
& A_{h}^{e}=\left(k_{0}\right)^{2}-\epsilon^{e}\left(\xi_{h}^{e}, \psi_{h}^{e}\right)^{2}-\frac{2 e \alpha_{k}^{2} E_{k}}{\left(k_{0}-\mu\right)-e E_{k}}\left(\psi_{h}^{e}\right)^{2}, \\
& B_{h}^{e}=\xi_{h}^{-e} \psi_{h}^{e}-\xi_{h}^{e} \psi_{h}^{-e}+\frac{2 e E_{k}}{\left(k_{0}-\mu\right)+e E_{k}} \xi_{h}^{e} \psi_{h}^{-e},
\end{aligned}
$$

using the notation:

$$
\epsilon^{ \pm}(\xi, \psi)=\left[\left(E_{k} \mp \mu\right)^{2}+\xi^{2}+\alpha_{k}^{2} \psi^{2}\right]^{1 / 2}
$$

\footnotetext{
${ }^{3}$ see Appendix A for a short review of the Nambu-Gorkov formalism.
} 
Using the $2 \times 2$ matrix representation of $P_{h}^{e}$ and $Q_{h}^{e}$ given above:

$$
\Omega^{-1}=\sum_{e, h=-1,1} \frac{1}{\operatorname{det} \Omega_{h}}\left(A_{h}^{-e} P_{h}^{e}(k)-B_{h}^{e} Q_{h}^{e}(k)\right),
$$

where:

$$
\begin{aligned}
\operatorname{det} \Omega_{h} & =k_{0}^{4}-2 k_{0}^{2}\left(E_{k}^{2}+\mu^{2}+\left(\xi_{h}^{+}\right)^{2}+\left(\xi_{h}^{-}\right)^{2}+\alpha^{2}\left(\psi_{h}^{+}\right)^{2}+\alpha^{2}\left(\psi_{h}^{-}\right)^{2}\right) \\
& +2 E_{k} k_{0}\left(\left(\psi_{h}^{+}\right)^{2}-\left(\psi_{h}^{-}\right)^{2}\right) \\
& +\left[\left(E_{k}-\mu\right)^{2}\left(E_{k}+\mu\right)^{2}+\left(E_{k}+\mu\right)^{2}\left(\xi_{h}^{+}\right)^{2}+\left(E_{k}-\mu\right)^{2}\left(\xi_{h}^{-}\right)^{2}\right. \\
& \left.-\alpha^{2}\left(E_{k}-\mu\right)\left(E_{k}+\mu\right)\left(\left(\psi_{h}^{+}\right)^{2}-\left(\psi_{h}^{-}\right)^{2}\right)+\left(\xi_{h}^{+} \xi_{h}^{-}+\alpha^{2} \psi_{h}^{+} \psi_{h}^{-}\right)^{2}\right]
\end{aligned}
$$

and the definition:

$$
E_{k}=\sqrt{|\vec{k}|^{2}+m^{2}}
$$

has been used. The poles of the quasiparticle propagator are given by the roots of this quartic equation in $k_{0}$. The roots have well known analytic forms that are nonetheless quite complicated and are not necessary for the purposes of this paper.

From (34) is it clear that:

$$
G^{+}(k)=\Omega^{-1}\left(\left(k_{0}-\mu\right) \gamma^{0}-\gamma \cdot \vec{k}+M\right),
$$

and therefore the general quasiparticle propagator is:

$$
\begin{aligned}
& G^{+}(k)= \\
& \sum_{e, h=-1,1} \frac{\left.\alpha\left(e k_{0}-e \mu+E_{k}\right)\right)\left(k_{0}^{2}-\left(\omega_{1}\right)_{h}^{e}\right)-\alpha\left(e k_{0}-e \mu-E_{k}\right)\left(\beta v_{h}^{e}\right) \psi_{h}^{-e}}{\operatorname{det} \Omega_{h}} P_{h}^{e} \\
& -\sum_{e, h=-1,1} \frac{\beta / \alpha\left(e k_{0}-e \mu-E_{k}\right)\left(k_{0}^{2}-\left(\omega_{2}\right)_{h}^{e}\right)+\alpha\left(e k_{0}-e \mu-E_{k}\right) \eta_{h}^{e} \psi_{h}^{-e}}{\operatorname{det} \Omega_{h}} Q_{h}^{e} .
\end{aligned}
$$

where:

$$
\begin{aligned}
\left(\omega_{1}\right)_{h}^{e} & =\left(E_{k}+e \mu\right)^{2}+\xi_{h}^{-e} \chi_{h}^{-e} \\
\left(\omega_{2}\right)_{h}^{e} & =\left(E_{k}+e \mu\right)^{2}+\frac{1}{\beta} \xi_{h}^{-e} \phi_{h}^{-e}
\end{aligned}
$$


and:

$$
\begin{aligned}
\phi_{h}^{e} & =\beta \xi_{h}^{e}+\alpha^{2} \psi_{h}^{e}, \\
\chi_{h}^{e} & =\xi_{h}^{e}-\beta \psi_{h}^{e}, \\
v_{h}^{e} & =\beta \xi_{h}^{e}+\alpha^{2} \psi_{h}^{-e}, \\
\eta_{h}^{e} & =\xi_{h}^{e}-\beta \psi_{h}^{-e} .
\end{aligned}
$$

This propagator could be diagonalized but the diagonalization depends on $k_{0}$ and $E_{k}$ and is quite complicated. It is not necessary to diagonalize the complete propagator for the purposes of this paper.

Assuming that only the $\gamma_{5}$ condensate contributes, this corresponds to the relations:

$$
\xi_{h}^{e} \equiv \chi_{h}^{e} \equiv \beta \phi_{h}^{e}=e h \xi_{+}^{+}, \quad \psi_{h}^{e} \equiv 0, \quad v_{h}^{e} \equiv \beta \xi_{h}^{e}, \quad \eta_{h}^{e} \equiv \xi_{h}^{e},
$$

and it can be shown that:

$$
\begin{aligned}
G^{+} & =\sum_{e, h=-1,1} \frac{k_{0}+e\left(E_{k}-e \mu\right)}{k_{0}^{2}-\left(E_{k}-e \mu\right)^{2}-\left(\xi_{+}^{+}\right)^{2}} P_{h}^{e} \gamma_{0} \\
& =\sum_{e=-1,1} \frac{k_{0}+e\left(E_{k}-e \mu\right)}{k_{0}^{2}-\left(E_{k}-e \mu\right)^{2}-\left(\xi_{+}^{+}\right)^{2}} \Lambda^{e} \gamma_{0},
\end{aligned}
$$

which agrees with the quark propagator derived in [22] using this ansatz. While this ansatz is a good first approximation, it will be shown that the gap equation will not close under this ansatz and the general quark propagator is the more complex form shown in (41).

The analysis up until this point generalizes to perturbation theory. In what follows the analysis will be restricted to NJL models where things simplify considerably. With foresight define:

$$
\begin{aligned}
\psi_{h}^{e} & =h \frac{\beta}{\alpha} \Delta_{3}, \\
\xi_{h}^{e} & =h\left(e \Delta_{1}-\alpha \Delta_{3}\right),
\end{aligned}
$$

where $\Delta_{1}$ is the usual CFL condensate and $\Delta_{3}$ is a new condensate and the gap matrix is now given by:

$$
\Delta=\Delta_{1} \gamma_{5}+\Delta_{3} \gamma_{5} \gamma_{0}
$$


In this case:

$$
\operatorname{det} \Omega_{+}=\operatorname{det} \Omega_{-}=\left(k_{0}^{2}-\left(\varepsilon^{+}\right)^{2}\right)\left(k_{0}^{2}-\left(\varepsilon^{-}\right)^{2}\right),
$$

where the poles of the quasiparticle propagator are given by:

$$
\left(\varepsilon^{ \pm}\right)^{2}=E_{k}^{2}+\mu^{2}+\Delta_{1}^{2}+\Delta_{3}^{2} \pm \sqrt{\left(2 E_{k} \mu+2 \alpha \Delta_{1} \Delta_{3}\right)^{2}+4 \beta^{2} \Delta_{3}^{2}\left(E_{k}^{2}+\Delta_{1}^{2}\right)} .
$$

Note that $\varepsilon^{ \pm}$are distinct from the $\epsilon^{ \pm}$defined in (36) and are used exclusively in what follows. These dispersion relations can be shown to agree with those stated in [21].

The poles of the propagator give the dispersion relations for quasiparticles, $\left(-\varepsilon^{-}\right)$, quasi-antiparticles $\left(-\varepsilon^{+}\right)$, quasiparticle holes $\left(\varepsilon^{-}\right)$and quasiantiparticles holes $\left(\varepsilon^{+}\right)$. The physical manifestation of the condensates is a gap in the quasiparticle spectrum between the maximum of the quasiparticle branch and the minimum of the quasiparticle hole branch:

$$
\varphi=\left(\left.\varepsilon^{-}\right|_{\min }\right)-\left(-\left.\varepsilon^{-}\right|_{\max }\right)=\left.2 \varepsilon^{-}\right|_{\min } .
$$

This means that exciting a quasiparticle requires a minimum energy, $\varphi$.

In the massless limit $\Delta_{3}=0, \alpha=0, E_{k}=|\vec{k}|$ and therefore the $\varepsilon^{ \pm}$reduce to $\epsilon^{\mp}$ defined in [27]. In this case the gap is simply $\varphi=2 \Delta_{1}$ at $k=\mu$.

In the massive case, $\Delta_{3}$ alters the dispersion relations. The minimum of the quasiparticle hole spectrum occurs at:

$$
E_{k}=\sqrt{\mu^{2}+\Delta_{1}^{2}+\Delta_{3}^{2}-\frac{2 m \mu \Delta_{1}+\left(\Delta_{1}^{2}-m^{2}\right) \Delta_{3}}{\Delta_{3}^{2}+\mu^{2}}},
$$

and leads to a gap:

$$
\varphi=2 \frac{\mu \Delta_{1}-m \Delta_{3}}{\sqrt{\mu^{2}+\Delta_{3}^{2}}} .
$$

For $\Delta_{3}=0$ the minimum occurs at $E_{k}=\mu$ and leads to a gap of $\varphi=2 \Delta_{1}$. When $\Delta_{3}=0$ is taken into account the minimum is shifted to a slightly lower value and the gap increases or decreases depending on the sign of $\Delta_{3}$. Further discussion of the dispersion relations and the gap will be given in subsequent sections in terms of specific solutions. 
In the NJL model the full propagator is;

$$
\begin{aligned}
& G^{+}(k)= \\
& \sum_{e, h=-1,1} \frac{\left.\alpha e\left(k_{0}-\mu+e E_{k}\right)\right)\left(k_{0}^{2}-\left(\omega_{1}\right)_{+}^{e}\right)-e\left(k_{0}-\mu-e E_{k}\right) \beta^{3} \Delta_{1} \Delta_{3}}{\left(k_{0}^{2}-\left(\varepsilon^{+}\right)^{2}\right)\left(k_{0}^{2}-\left(\varepsilon^{-}\right)^{2}\right)} P_{h}^{e} \\
& -\frac{\beta / \alpha\left(e k_{0}-e \mu-E_{k}\right)\left(k_{0}^{2}-\left(\omega_{2}\right)_{+}^{e}\right)+\left(k_{0}-\mu-e E_{k}\right)\left(\Delta_{1}-e \Delta_{3} / \alpha\right) \Delta_{3}}{\left(k_{0}^{2}-\left(\varepsilon^{+}\right)^{2}\right)\left(k_{0}^{2}-\left(\varepsilon^{-}\right)^{2}\right)} Q_{h}^{e},
\end{aligned}
$$

where:

$$
\begin{aligned}
& \left(\omega_{1}\right)_{h}^{e}=\left(E_{k}+e \mu\right)^{2}+\left(\Delta_{1}+e \alpha \Delta_{3}\right)\left(\Delta_{1}+e \Delta_{3} / \alpha\right) \\
& \left(\omega_{2}\right)_{h}^{e}=\left(E_{k}+e \mu\right)^{2}+\left(\Delta_{1}+e \alpha \Delta_{3}\right) \Delta_{1} .
\end{aligned}
$$

The quasiparticle propagator can now be used in the mean field gap equation 27 (A6):

$$
\Delta(k)=-i g^{2} \int \frac{d^{4} q}{(2 \pi)^{4}} \sum_{A, B=1 . . N_{c}^{2}-1} \bar{\Gamma}_{\mu}^{A} D_{A B}^{\mu \nu}(k-q) G_{0}^{-}(q) \Delta(q) G^{+}(q) \Gamma_{\nu}^{B},
$$

where $D_{A B}^{\mu \nu}(k-q)$ is the gluon propagator, $\Gamma_{\nu}^{B}$ is the interaction vertex, and(see Appendix A):

$$
\bar{\Gamma}=C \Gamma^{T} C^{-1} .
$$

In the following sections results are presented for two different types of four fermion interactions. The next section presents results for the scalar NJL model. Section V presents results for an NJL model which has the color structure of single gluon exchange. These models give identical results if $\Delta_{3}$ is neglected but differ quite significantly if it is included.

One of the main results of this section is the general quasiparticle propagator (41) which could be used in a perturbation theory analysis of the gap equation. The second main result of this section is the quasiparticle propagator for an NJL model (58) which is used in the rest of this paper. The poles of the quasiparticle propagator (54) are particularly important for this analysis. Finally the definition of the gap in terms of the condensates (57) is another result which will be important in the rest of this paper. 


\section{Scalar NJL Model}

The simplest possible four fermion interaction, motivated by the effective Lagrangian approach, is to take the interaction vertex to be $\Gamma_{\mu}^{A}=i$ and:

$$
g^{2} D_{A B}^{\mu \nu} \rightarrow \frac{2 \pi^{2}}{N_{c}^{2}-1} G \delta^{\mu \nu} \delta_{A B}
$$

giving the matrix gap equation:

$$
\Delta(k)=8 i \pi^{2} G \int \frac{d^{4} q}{(2 \pi)^{4}} G_{0}^{-}(q) \Delta(q) G^{+}(q) .
$$

Acting on both sides of this equation with the operators $\gamma_{5}, \gamma_{5} \gamma_{0}$ and tracing over the spinor indices one can obtain the gap equations for $\Delta_{1}$ and $\Delta_{3}$ respectively. The color-flavor structure of the gap matrix can almost be ignored because the propagators do not have any color flavor structure and therefore the same factor will occur on both sides of the equation. If one is working in the degenerate three flavor case there is a slight complication to even the zero mass gap equation because of the existence of the $\Delta_{66}$ condensate. This complication is ignored in this paper so this analysis is an approximation in the three flavor case.

In the two flavor case there is only one color-flavor structure to be concerned about and this analysis is exact. This applies even to the case of the 2SC color superconducting phase in the physical 3 flavor case as condensates involving the third color and flavor will simply decouple from the condensates of interest.

The coupled gap equations are:

$$
\begin{aligned}
\Delta_{1} & =8 i G \pi^{2} \int \frac{d^{4} q}{(2 \pi)^{4}} \frac{2 E_{q} \mu \alpha \Delta_{3}+\Delta_{1}\left(q_{0}^{2}-E_{q}^{2}-\Delta_{1}^{2}+\Delta_{3}^{2}-\mu^{2}\right)}{\left(q_{0}^{2}-\left(\varepsilon^{+}\right)^{2}\right)\left(q_{0}^{2}-\left(\varepsilon^{-}\right)^{2}\right)} \\
\Delta_{3} & =-8 i G \pi^{2} \int \frac{d^{4} q}{(2 \pi)^{4}} \\
& \frac{2 E_{q} \mu \alpha \Delta_{1}+\Delta_{3}\left(q_{0}^{2}+\Delta_{1}^{2}-\mu^{2}\right)+\Delta_{3}\left(\beta^{2}-\alpha^{2}\right)\left(E_{q}^{2}-\Delta_{3}^{2}\right)}{\left(q_{0}^{2}-\left(\varepsilon^{+}\right)^{2}\right)\left(q_{0}^{2}-\left(\varepsilon^{-}\right)^{2}\right)}
\end{aligned}
$$

where terms linear in $q_{0}$ have been dropped since they will cancel out on integration over $q_{0}$ from $-\infty$ to $\infty$. The dependence of the condensate on momentum has been dropped since the right hand side of the gap equations are independent of $\vec{k}$. 
Acting on (64) with the operators $\gamma_{0}$ and the identity and taking the trace leads to vanishing of the right hand side which is consistent with the assumption that $\Delta_{4}$ and $\Delta_{8}$ are zero. Acting with any of the other operators involving $\gamma \cdot \vec{k}$ will lead to a term involving $\hat{q} \cdot \hat{k}$ which will vanish by symmetry under the angular integration.

Evaluation of these equations can be facilitated by the analytic continuation $q_{0} \rightarrow-i q_{4}$. The $q_{4}$ integration is then done by contour integration closing the contour in the upper half plane and picking up the poles at $i \varepsilon^{+}$ and $i \varepsilon^{-}$. The angular integrals can be done trivially giving:

$$
\begin{aligned}
\Delta_{1} & =G \int d q q^{2}\left(\frac{\Delta_{1}}{\varepsilon^{+}}+\frac{\Delta_{1}}{\varepsilon^{-}}+\frac{4\left(m \mu+\Delta_{1} \Delta_{3}\right) \Delta_{3}}{\left(\left(\varepsilon^{+}\right)^{2}-\left(\varepsilon^{-}\right)^{2}\right) \varepsilon^{+}}-\frac{4\left(m \mu+\Delta_{1} \Delta_{3}\right) \Delta_{3}}{\left(\left(\varepsilon^{+}\right)^{2}-\left(\varepsilon^{-}\right)^{2}\right) \varepsilon^{-}}\right), \\
\Delta_{3} & =G \int d q q^{2}\left(-\frac{\Delta_{3}}{\varepsilon^{+}}-\frac{\Delta_{3}}{\varepsilon^{-}}-\frac{4 m \mu \Delta_{1}}{\left(\left(\varepsilon^{+}\right)^{2}-\left(\varepsilon^{-}\right)^{2}\right) \varepsilon^{+}}+\frac{4 m \mu \Delta_{1}}{\left(\left(\varepsilon^{+}\right)^{2}-\left(\varepsilon^{-}\right)^{2}\right) \varepsilon^{-}}\right. \\
& \left.-\frac{4\left(q^{2}+\Delta_{1}^{2}\right) \Delta_{3}}{\left(\left(\varepsilon^{+}\right)^{2}-\left(\varepsilon^{-}\right)^{2}\right) \varepsilon^{+}}+\frac{4\left(q^{2}+\Delta_{1}^{2}\right) \Delta_{3}}{\left.\left(\left(\varepsilon^{+}\right)^{2}-\left(\varepsilon^{-}\right)\right)^{2}\right)}\right) .
\end{aligned}
$$

If one assumes $\Delta_{3}=0$ the first equation reduces to the gap equation solved in [22]. The second equation does not vanish exactly under this assumption. If one takes $m=0$ and $\Delta_{3}=0$ the second equation is trivially satisfied and the first equation becomes the gap equation for massless quarks.

The range of integration for $q$ is not infinite since the NJL model is a four-fermion interaction model and must have an UV cutoff, $\Lambda$, which is left arbitrary except for the restriction that it be greater than $\mu$.

An approximate solution of the gap equation for $m=0$ is given by [5]:

$$
\Delta_{1}^{(0)} \equiv \Delta_{1}(m=0) \approx 2 \sqrt{\Lambda^{2}-\mu^{2}} \exp \left[\frac{\Lambda^{2}-3 \mu^{2}}{2 \mu^{2}}\right] \exp \left[-\frac{1}{2 G \mu^{2}}\right] \text {. }
$$

Expanding the integrands of (67) and (68) in $m$ one can obtain approximate solutions for the condensates:

$$
\begin{aligned}
\Delta_{1}(m) \approx & \Delta_{1}^{(0)}\left[1-m^{2}\left\{\frac{1-G\left(\Lambda^{2}-\mu^{2}\right)}{4 G \mu^{4}}-\frac{1}{2\left(\Lambda^{2}-\mu^{2}\right)}+A \frac{1-G\left(\Lambda^{2}+\mu^{2}\right)}{2 G \mu^{4}}\right\}\right. \\
& +\frac{1}{2} m^{4}\left\{\frac{1-G\left(\Lambda^{2}-\mu^{2}\right)}{4 G \mu^{4}}-\frac{1}{2\left(\Lambda^{2}-\mu^{2}\right)}+A \frac{1-G\left(\Lambda^{2}+\mu^{2}\right)}{2 G \mu^{4}}\right\}^{2}
\end{aligned}
$$




$$
\begin{aligned}
+ & m^{4}\left\{\frac{3\left(G\left(\Lambda^{2}-\mu^{2}\right)-1\right)}{16 G \mu^{6}}-\frac{1}{4\left(\Lambda^{2}-\mu^{2}\right)^{2}}+\frac{\log \left[\frac{\Lambda}{\mu}\right]}{8 \mu^{4}}\right\} \\
+ & \left.A m^{4}\left\{\frac{\left(G\left(\Lambda^{2}+\mu^{2}\right)-1\right)}{4 G \mu^{6}}+\frac{A\left(G \Lambda^{2}-1\right)}{2 G \mu^{6}}-\frac{\log \left[\frac{\Lambda}{\mu}\right]}{2 \mu^{4}}\right\}\right], \\
\Delta_{3}(m) & \approx A \frac{m}{\mu} \Delta_{1}(m),
\end{aligned}
$$

where:

$$
A=1-\frac{3 \Lambda^{2}-\mu^{2}}{3 \Lambda^{2}-7 \mu^{2}+3 \mu^{2} \log \left[\left(\Delta_{1}^{(0)}\right)^{2} / 4\left(\Lambda^{2}-\mu^{2}\right)\right]} .
$$

In order to verify the accuracy of the approximate solutions the exact gap equations were also solved numerically using Mathematica ${ }^{\complement}$ for comparison. These approximations are somewhat complicated due to their generality and range of applicability so some general comments are in order.

The solution for $\Delta_{3}$ is linear in $m / \mu$ for small values of $\mathrm{m}$ with slope $A$ ranging from $A=1$ for $\Delta_{1}^{(0)}=0$ to $A=0$ for $\Delta_{1}^{(0)}=\frac{2}{e} \sqrt{\Lambda^{2}-\mu^{2}}$.

The solution for $\Delta_{1}(m)$ is an expansion in $\frac{m^{2}}{\mu^{2}}$ to order $\mathcal{O}\left(m^{4} / \mu^{4}\right)$. Factors of $1 / G \mu^{2}$ in some terms are actually enhancement factors as can be seen by taking typical values of $G=6 \mathrm{GeV}^{-2} / \pi^{2}$ and $\mu=500 \mathrm{MeV}$ giving a value of $1 / G \mu^{2} \approx 6.5$. Expanding only to $\mathcal{O}\left(\mathrm{m}^{2} / \mu^{2}\right)$ :

$\Delta_{1}(m)=\Delta_{1}^{(0)}\left[1-m^{2}\left\{\frac{1-G\left(\Lambda^{2}-\mu^{2}\right)}{4 G \mu^{4}}-\frac{1}{2\left(\Lambda^{2}-\mu^{2}\right)}+A \frac{1-G\left(\Lambda^{2}+\mu^{2}\right)}{2 G \mu^{4}}\right\}\right]$.

it can be shown that the first and last terms dominate and are of the same order of magnitude. This means that neglecting the effect of $\Delta_{3}$ by setting $A=0$ will have a non-trivial effect on the solution for $\Delta_{1}(m)$. The order $\mathcal{O}\left(m^{4} / \mu^{4}\right)$ terms are less instructive and are only included in order that the approximation is accurate for a wider range of $m, \mu, \Lambda$ and $G$.

The accuracy of this approximation for a range of four fermion couplings, $G$, is illustrated in Figure (11). Examining the limiting case $G \rightarrow 0$ which corresponds to $\Delta_{1}^{(0)} \rightarrow 0$, it can be seen that effect of the quark mass becomes increasingly important. Conversely, as $\Delta_{1}^{(0)}$ increases the effect of the quark mass becomes less significant. Results obtained by varying $\Lambda$ or $\mu$ have exactly the same behavior and also agree well with the exact numerical results. 


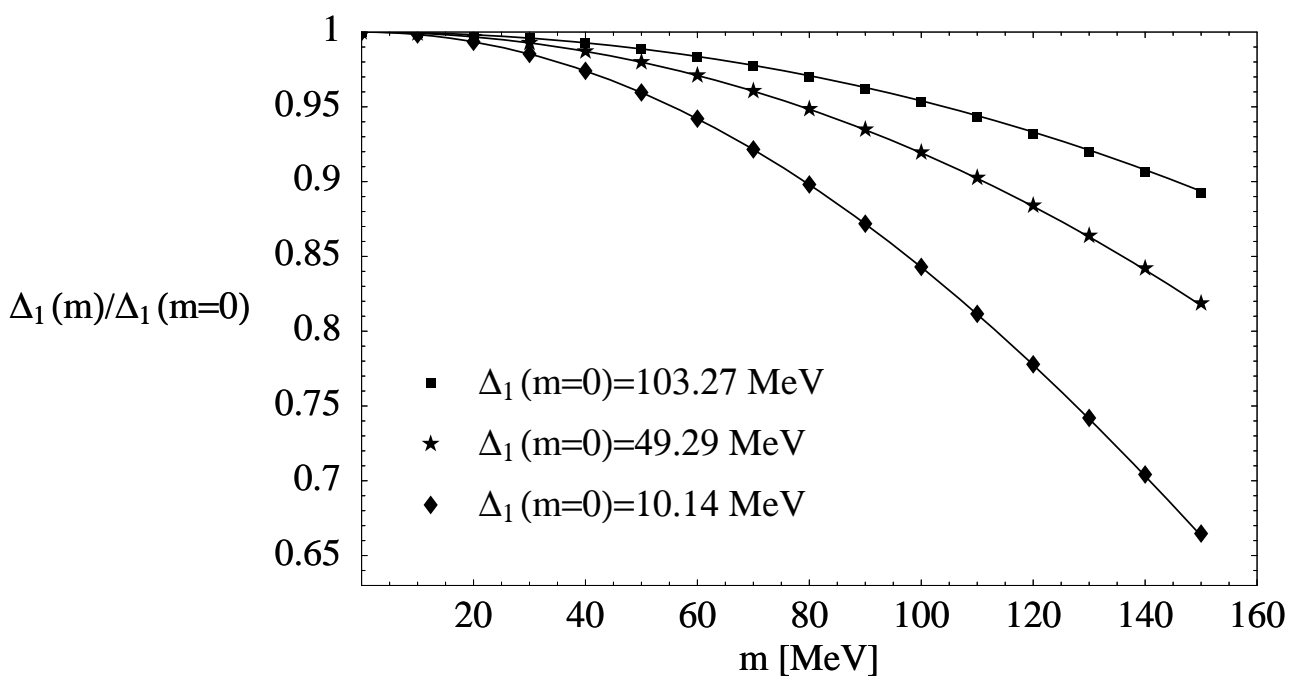

Figure 1: $\Delta_{1}(m)$ for $\mu=500 \mathrm{MeV}$ and $\Lambda=2 \mu$ in the scalar NJL model for (from bottom to top) $G \pi^{2}\left[\mathrm{GeV}^{-2}\right]=3.5,4.875,6$. Analytic approximations and exact numerical results are shown for comparison.

The solutions for $G=3.5 \mathrm{GeV}^{-2} / \pi^{2}$ and $G=6 \mathrm{GeV}^{-2} / \pi^{2}$ which correspond to $\Delta_{1}^{(0)}=10.14 \mathrm{MeV}$ and $\Delta_{1}^{(0)}=103.27 \mathrm{MeV}$ are shown in Figures (2) and (3) with $\mu=500 \mathrm{MeV}$. Also shown in Figure (2) are the solutions where $\Delta_{3}$ is neglected for comparison. The effect on $\Delta_{1}(\mathrm{~m})$ of including $\Delta_{3}$ is an increase in the mass dependence compared to the solution neglecting $\Delta_{3}$. The effect is more pronounced for smaller values of $\Delta_{1}^{(0)}$, but is still significant at $\Delta_{1}^{(0)} \approx 100 \mathrm{MeV}$. Notice that the relative size of $\Delta_{3}$ with respect to $\Delta_{1}$ is very similar in both cases.

As discussed in the previous section, inclusion of $\Delta_{3}$ alters the dispersion relations and the gap, $\varphi$. The gap between the quasiparticle hole branch and quasiparticle branch in this case is not simply $\varphi=2 \Delta_{1}(m)$ but is given by (57). The value of the gap is plotted for the same choices of $G$ presented above in Figure (4) with and without $\Delta_{3}$. The effect on the gap is larger than the effect on $\Delta_{1}$ alone because $\Delta_{3}$ affects $\varphi$ directly in (57) as well as indirectly through $\Delta_{1}$. The dispersion relation for the quasiparticle holes is not appreciably altered. 

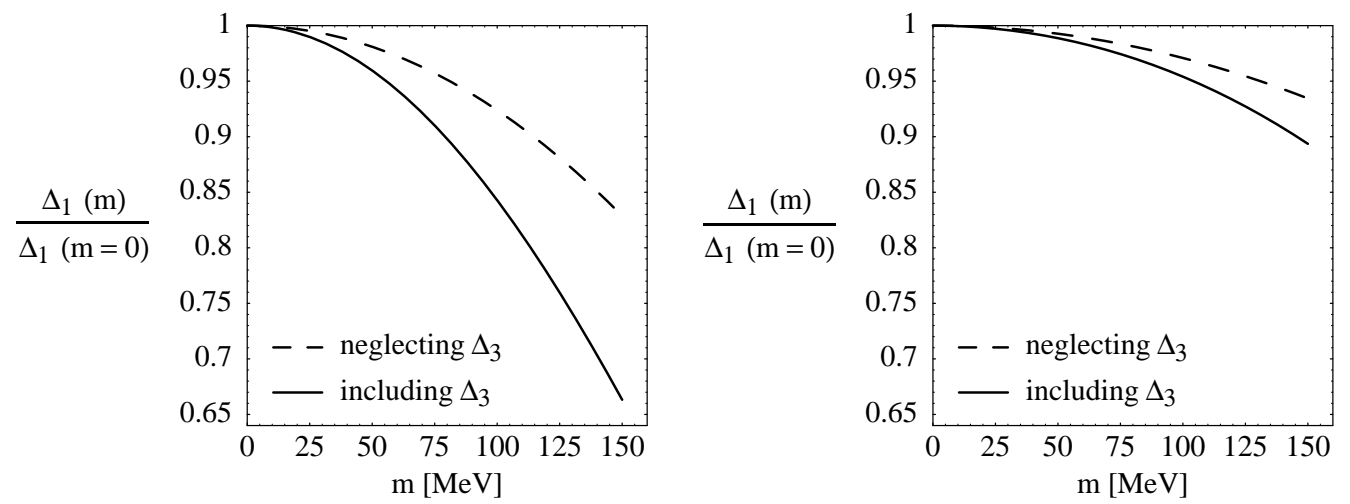

Figure 2: $\Delta_{1}(m)$ for $\mu=500 \mathrm{MeV}$ and $\Lambda=2 \mu$ in the scalar NJL model. The graph on the left is for $G=3.5 \mathrm{GeV}^{-2} / \pi^{2}$ and $\Delta_{1}^{(0)}=10.14 \mathrm{MeV}$. The graph on the right is for $G=6 \mathrm{GeV}^{-2} / \pi^{2}$ and $\Delta_{1}^{(0)}=103.27 \mathrm{MeV}$. Solutions are shown neglecting and including $\Delta_{3}$. 


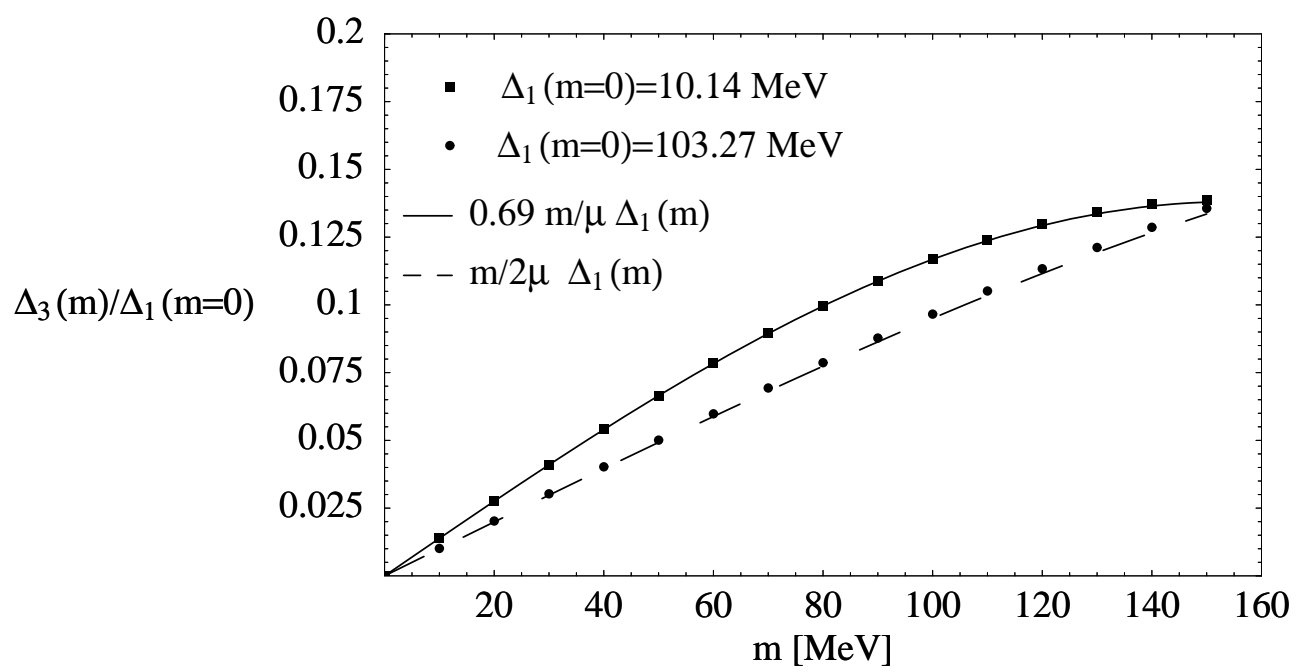

Figure 3: $\Delta_{3}(m)$ for $\mu=500 \mathrm{MeV}$ and $\Lambda=2 \mu$ in the scalar NJL model. Four fermion coupling constants $G=3.5 \mathrm{GeV}^{-2} / \pi^{2}$ and $G=6 \mathrm{GeV}^{-2} / \pi^{2}$ were used and give solutions with $\Delta_{1}^{(0)}=10.14 \mathrm{MeV}$ and $\Delta_{1}^{(0)}=103.27$ $\mathrm{MeV}$. Analytic approximations and exact numerical results are shown for both cases. 

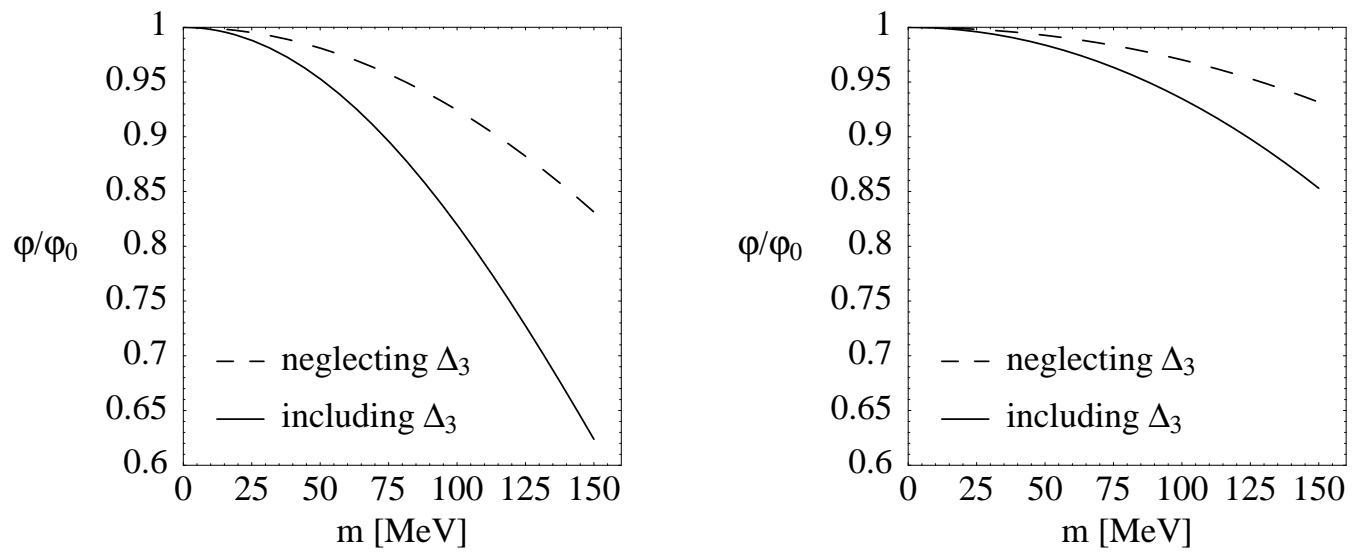

Figure 4: Plot of $\varphi / \varphi_{0}$ for the scalar NJL model including and neglecting $\Delta_{3}$ for different values of $\Delta_{1}^{(0)}$. The graph on the left is for $G=3.5 \mathrm{GeV}^{-2} / \pi^{2}$ and $\Delta_{1}^{(0)}=10.14 \mathrm{MeV}$. The graph on the right is for $G=6 \mathrm{GeV}^{-2} / \pi^{2}$ and $\Delta_{1}^{(0)}=103.27 \mathrm{MeV}$. 


\section{NJL Model motivated by Single Gluon Ex- change}

The NJL interaction which has the structure of single gluon exchange has the interaction vertices:

$$
\Gamma_{\mu}^{A}=\gamma_{\mu} \frac{\lambda_{c}^{A}}{2}, \quad \bar{\Gamma}_{\mu}^{A}=-\gamma_{\mu}\left(\frac{\lambda_{c}^{A}}{2}\right)^{T},
$$

and:

$$
g^{2} D_{A B}^{\mu \nu} \rightarrow 3 \pi^{2} G g^{\mu \nu} \delta_{A B},
$$

giving the gap equation:

$$
\Delta(k)=-2 i G \int \frac{d^{4} q}{(2 \pi)^{4}} \gamma_{\nu} G_{0}^{-}(q) \Delta(q) G^{+}(q) \gamma^{\nu},
$$

using the equations:

$$
\left(\lambda^{A}\right)_{i j}^{T} \lambda_{j k}^{B} \lambda_{k l}^{A}=-\frac{8}{3} \lambda_{i l}^{B} \quad \text { assuming } \lambda^{B} \text { is antisymmetric. }
$$

Multiplying each side of equation $(\sqrt{76})$ by $\gamma_{5}$ and $\gamma_{5} \gamma_{0}$, tracing over the Dirac indices and using the cyclicity of the trace and the relations:

$$
\begin{gathered}
\gamma_{\mu} \gamma^{5} \gamma^{\mu}=-4 \gamma^{5}, \\
\gamma_{\mu} \gamma^{5} \gamma^{0} \gamma^{\mu}=-\gamma^{5} \gamma_{\mu} \gamma^{0} \gamma^{\mu}=2 \gamma^{5} \gamma^{0},
\end{gathered}
$$

and then applying all the other machinery of the last section, the coupled gap equations are obtained:

$$
\begin{aligned}
\Delta_{1} & =G \int d q q^{2}\left(\frac{\Delta_{1}}{\varepsilon^{+}}+\frac{\Delta_{1}}{\varepsilon^{-}}+\frac{4\left(m \mu+\Delta_{1} \Delta_{3}\right) \Delta_{3}}{\left(\left(\varepsilon^{+}\right)^{2}-\left(\varepsilon^{-}\right)^{2}\right) \varepsilon^{+}}-\frac{4\left(m \mu+\Delta_{1} \Delta_{3}\right) \Delta_{3}}{\left(\left(\varepsilon^{+}\right)^{2}-\left(\varepsilon^{-}\right)^{2}\right) \varepsilon^{-}}\right), \\
\Delta_{3} & =-\frac{G}{2} \int d q q^{2}\left(-\frac{\Delta_{3}}{\varepsilon^{+}}-\frac{\Delta_{3}}{\varepsilon^{-}}-\frac{4 m \mu \Delta_{1}}{\left(\left(\varepsilon^{+}\right)^{2}-\left(\varepsilon^{-}\right)^{2}\right) \varepsilon^{+}}+\frac{4 m \mu \Delta_{1}}{\left(\left(\varepsilon^{+}\right)^{2}-\left(\varepsilon^{-}\right)^{2}\right) \varepsilon^{-}}\right. \\
& \left.-\frac{4\left(q^{2}+\Delta_{1}^{2}\right) \Delta_{3}}{\left(\left(\varepsilon^{+}\right)^{2}-\left(\varepsilon^{-}\right)^{2}\right) \varepsilon^{+}}+\frac{4\left(q^{2}+\Delta_{1}^{2}\right) \Delta_{3}}{\left(\left(\varepsilon^{+}\right)^{2}-\left(\varepsilon^{-}\right)^{2}\right) \varepsilon^{-}}\right) .
\end{aligned}
$$


Notice that they are almost the same as the equations in the last section except for an overall factor of $-\frac{1}{2}$ in the second equation. Therefore the only change to the approximate solutions of the last section is:

$$
A=-\frac{1}{2}+\frac{3 \Lambda^{2}-4 \mu^{2}}{3 \Lambda^{2}-10 \mu^{2}+3 \mu^{2} \log \left[\left(\Delta_{1}^{(0)}\right)^{2} / 4\left(\Lambda^{2}-\mu^{2}\right)\right]} .
$$

For clarity the solution to order $\mathcal{O}\left(\mathrm{m}^{2} / \mu^{2}\right)$ is repeated here:

$$
\begin{gathered}
\Delta_{1}(m)=\Delta_{1}^{(0)}\left[1-m^{2}\left\{\frac{1-G\left(\Lambda^{2}-\mu^{2}\right)}{4 G \mu^{4}}-\frac{1}{2\left(\Lambda^{2}-\mu^{2}\right)}+A \frac{1-G\left(\Lambda^{2}+\mu^{2}\right)}{2 G \mu^{4}}\right\}\right], \\
\Delta_{3}(m)=A \frac{m}{\mu} \Delta_{1}(m) .
\end{gathered}
$$

The accuracy of this approximation for a range of these parameters is illustrated in Figures (5)-(6) by comparison with exact numerical solutions.

The main difference from the previous section is in the sign of A. $\Delta_{3}(m)$ is again linear in $m / \mu$ for small $\mathrm{m}$ but with a slope of $A \approx-\frac{1}{2}$ for small values of $\Delta_{1}^{(0)}$ and approaching 0 as $\Delta_{1}^{(0)}$ increases.

The first and third terms in the $\mathcal{O}\left(m^{2} / \mu^{2}\right)$ expansion of $\Delta_{1}(m)$ are of the same order of magnitude but in this case their signs differ. The resulting effect of the inclusion of $\Delta_{3}$ is to reduce the relative effect of the mass dependence.

The comments on the mass dependence as a function of the parameters $\mu$ and $G$ are unchanged from the previous section. For small values of $\Delta_{1}^{(0)}$ the effect is important and is less significant for increasing $\Delta_{1}^{(0)}$. This effect can be seen in Figure (5).

In the weak coupling limit, $G \rightarrow 0$, equations (83) and (84) are very similar to equations (37) and (34) of [24.

The mass dependence of $\Delta_{1}$ as a function of $\Lambda$ is different in this model than in the last section. In this case the last two terms of equation (83) decrease as $\Lambda$ approaches $\mu$ and although the first term still dominates, the relative mass dependence decreases with decreasing $\Lambda$. The effect is that the mass dependence is less significant for smaller $\Delta_{1}^{(0)}$ as can be seen in Figure (6).

The solutions for $G=3.5 \mathrm{GeV}^{-2} / \pi^{2}$ and $G=6 \mathrm{GeV}^{-2} / \pi^{2}$ which correspond to $\Delta_{1}(m=0)=10.14 \mathrm{MeV}$ and $\Delta_{1}(m=0)=103.27 \mathrm{MeV}$ are shown in Figures (7) and (8) with $\mu=500 \mathrm{MeV}$. The solutions where $\Delta_{3}$ is neglected are also shown in Figure (7) for comparison. The effect on $\Delta_{1}(m)$ 
of including $\Delta_{3}$ is a reduction in the relative effect compared to the solution neglecting $\Delta_{3}$. The effect is more pronounced for smaller values of $\Delta_{1}^{(0)}$, but is still nonneglegible at $\Delta_{1}^{(0)} \approx 100 \mathrm{MeV}$. Notice that the relative size of $\Delta_{3}$ with respect to $\Delta_{1}$ is very similar in both cases.

Inclusion of $\Delta_{3}$ alters the dispersion relations and the gap. The gap between the quasiparticle hole branch and quasiparticle branch is not simply $\varphi=2 \Delta_{1}$, but is again given by (57). The gap depends on $\Delta_{3}$ both explicitly and implicitly through its effect on $\Delta_{1}$. The value of the gap is plotted for the same choices of $G$ presented above in Figure (9) with and without $\Delta_{3}$. The effect on the gap is larger than the effect on $\Delta_{1}$ alone. The zeroth order approximation, $\Delta_{1}(m=0)$, is a better approximation to the gap than the solution where $\Delta_{3}$ is neglected. The dispersion relation for the quasiparticle holes is not appreciably altered.

Extending this analysis out to quark mass of $m=150 \mathrm{MeV}$ is partly for illustrative purposes but is also valuable for two other reasons.

First the analysis of 22] shows that in a coupled analysis of the superconducting (diquark) condensate and the axial condensate, constituent quark masses of the order of $100 \mathrm{MeV}$ for the light quarks are possible at $\mu \approx 400$ $\mathrm{MeV}$. For constituent quark masses of this order the results of our analysis show that the presence of a new condensate, $\Delta_{3}$ which they neglected in their ansatz will be relevant. Their ansatz is a reasonable first approximation as the new condensate and its effects on $\Delta_{1}$ are not large. However, the effect on the gap $\varphi$ itself is significant even for gaps of the order of $100 \mathrm{MeV}$. The full analysis requires the more general ansatz and the use of the general quasiparticle propagator. The effects of chiral symmetry breaking have not been taken into account in this paper as in [22, 21] but such an extension would not be difficult. The authors of [21] also found significant values for the constituent quark masses for $\mu \approx 400 \mathrm{MeV}$.

Second the methods used in this analysis can be extended to the physical case where the strange quark mass is of the order of $150 \mathrm{MeV}$. It is instructive therefore to carry out this analysis as a precursor to the physical case.

The two NJL models analyzed in this paper lead to quite different results when the full calculation is performed. In Figure (10) it is shown that the full calculation depends significantly on the type of NJL model used. For lower values of $\Delta_{1}^{(0)}$ the effect on the gap is almost a factor of 2 and is still significant even for $\Delta_{1}^{(0)}$ of the order of $100 \mathrm{MeV}$. The second NJL model analyzed is perhaps more physically motivated than the second but there is 


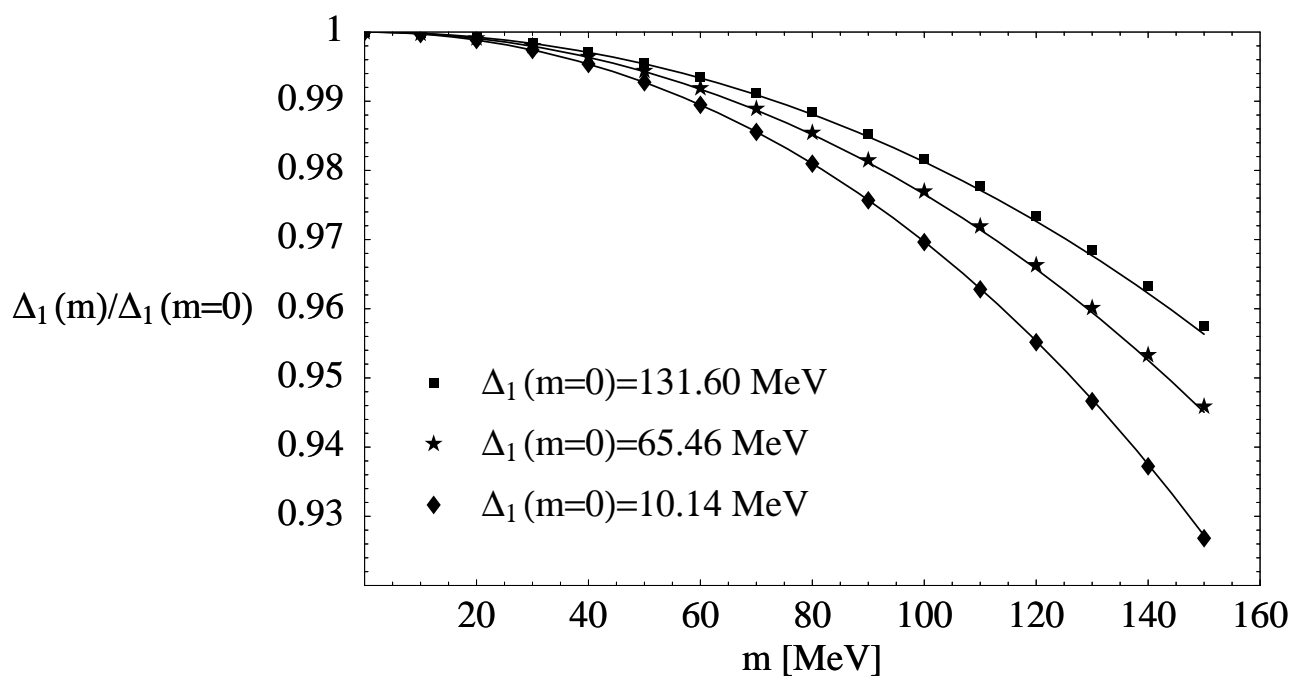

Figure 5: $\Delta_{1}(m)$ for $\mu=500 \mathrm{MeV}$ and $\Lambda=2 \mu$ in the NJL model with the color structure of single gluon exchange for (from bottom to top) $G \pi^{2}\left[\mathrm{GeV}^{-2}\right]=3.5,5.25,6.5$. Analytic approximations and exact numerical results are shown for comparison. 


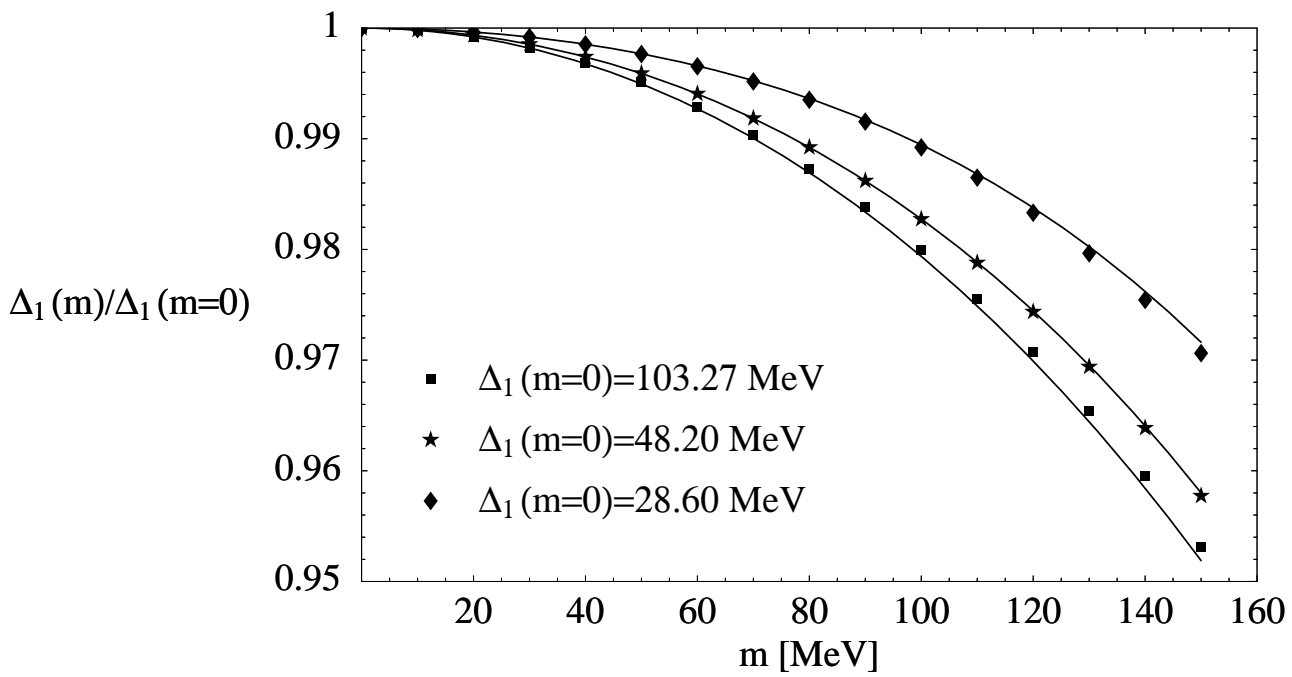

Figure 6: $\Delta_{1}(m)$ for $\mu=500 \mathrm{MeV}$ and $G=6 \mathrm{GeV}^{-2} / \pi^{2}$ in the NJL model with the color structure of single gluon exchange for (from bottom to top) $\Lambda=2 \mu, 1.7 \mu, 1.5 \mu$. Analytic approximations and exact numerical results are shown for comparison. 

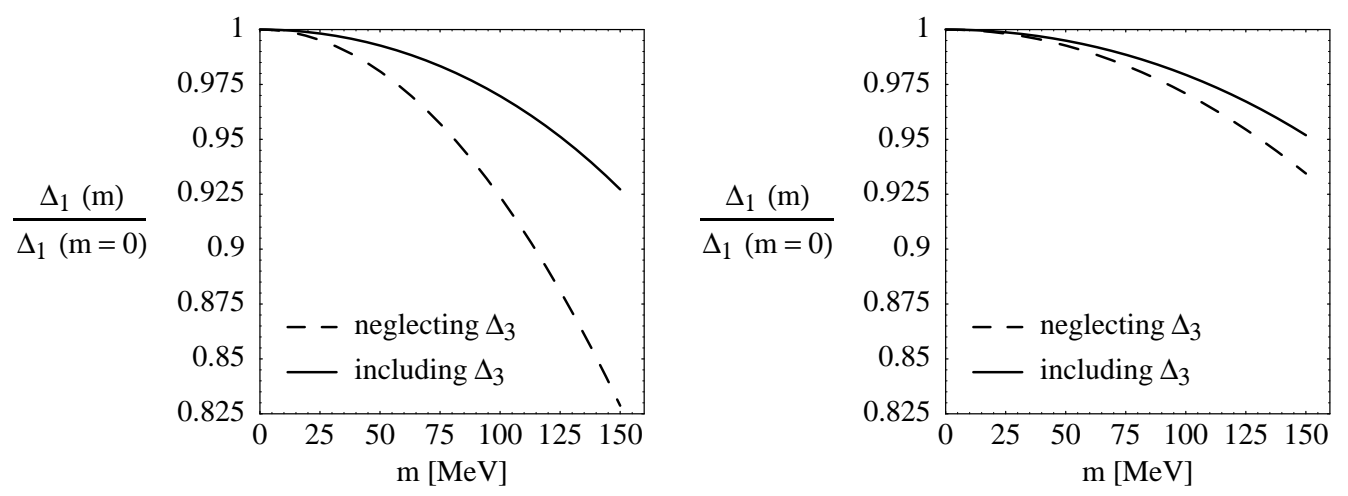

Figure 7: $\Delta_{1}(m)$ for $\mu=500 \mathrm{MeV}$ and $\Lambda=2 \mu$ in the NJL model with the color structure of single gluon exchange. The graph on the left is for $G=3.5 \mathrm{GeV}^{-2} / \pi^{2}$ and $\Delta_{1}^{(0)}=10.14 \mathrm{MeV}$. The graph on the right is for $G=6 \mathrm{GeV}^{-2} / \pi^{2}$ and $\Delta_{1}^{(0)}=10.14 \mathrm{MeV}$. Solutions are shown neglecting and including $\Delta_{3}$. 


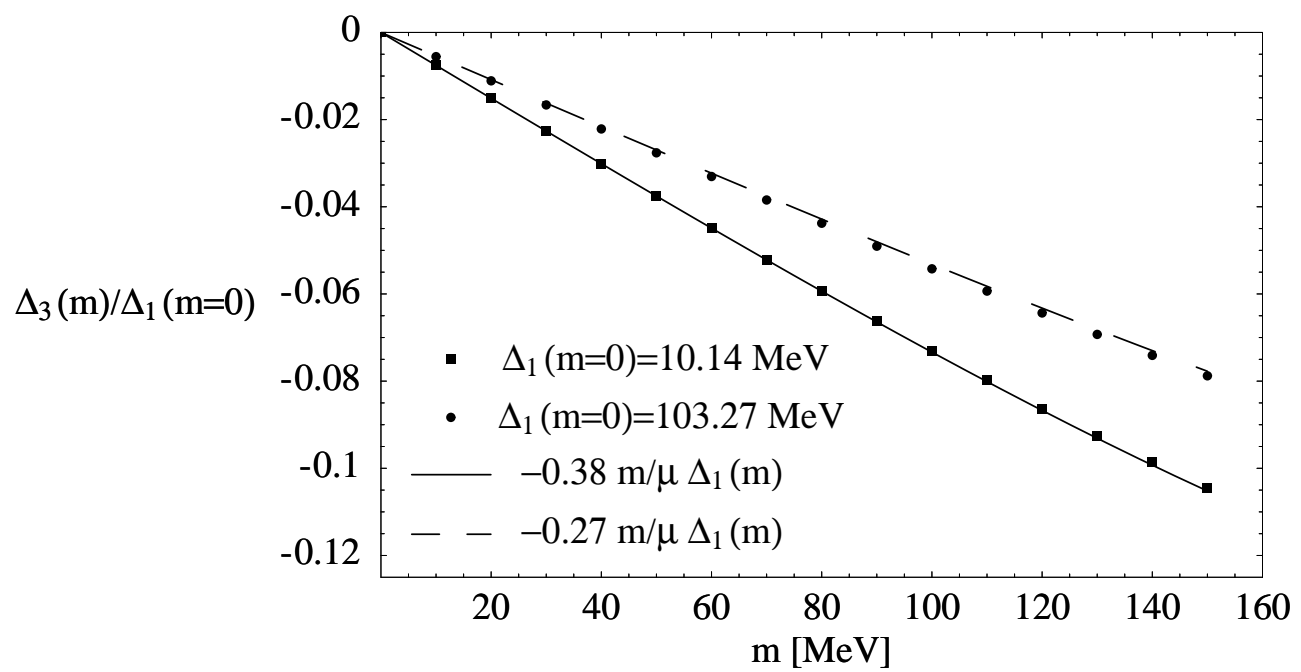

Figure 8: $\Delta_{3}(m)$ for $\mu=500 \mathrm{MeV}$ and $\Lambda=2 \mu$ in the NJL model with the color structure of single gluon exchange. Four fermion coupling constants $G=3.5 \mathrm{GeV}^{-2} / \pi^{2}$ and $G=6 \mathrm{GeV}^{-2} / \pi^{2}$ were used and give solutions with $\Delta_{1}^{(0)}=10.14 \mathrm{MeV}$ and $\Delta_{1}^{(0)}=103.27 \mathrm{MeV}$. Analytic approximations and exact numerical results are shown for both cases. 

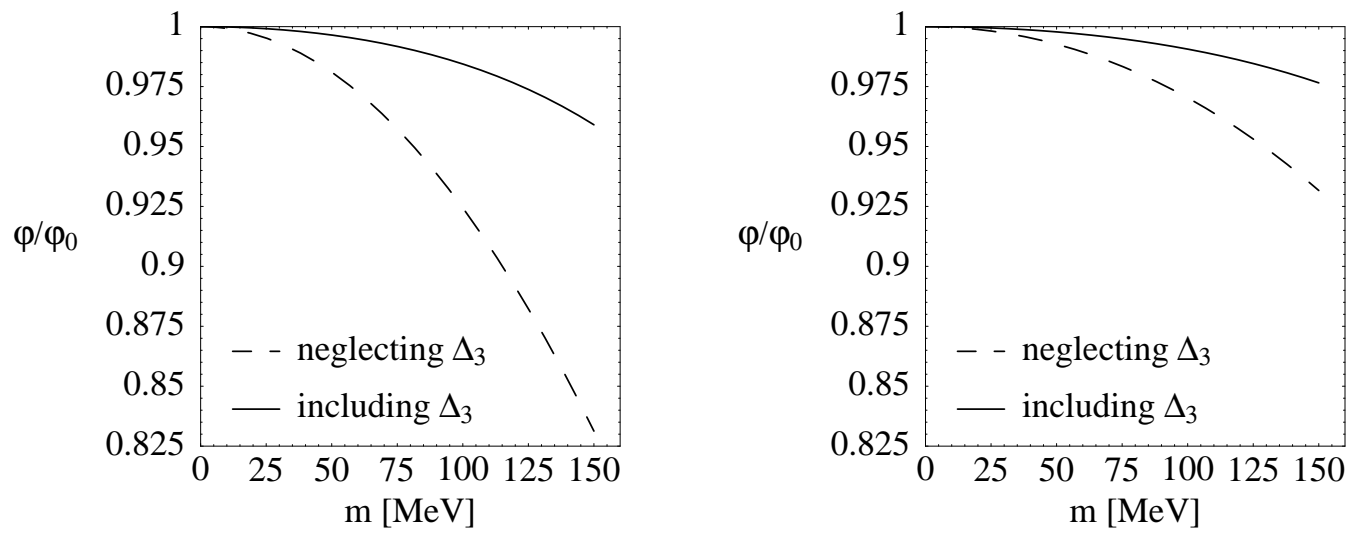

Figure 9: Plot of $\varphi / \varphi_{0}$ for the NJL model with color structure of single gluon exchange, including and neglecting $\Delta_{3}$ for different values of $\Delta_{1}^{(0)}$. The graph on the left is for $G=3.5 \mathrm{GeV}^{-2} / \pi^{2}$ and $\Delta_{1}^{(0)}=10.14 \mathrm{MeV}$. The graph on the right is for $G=6 \mathrm{GeV}^{-2} / \pi^{2}$ and $\Delta_{1}^{(0)}=103.27 \mathrm{MeV}$. 

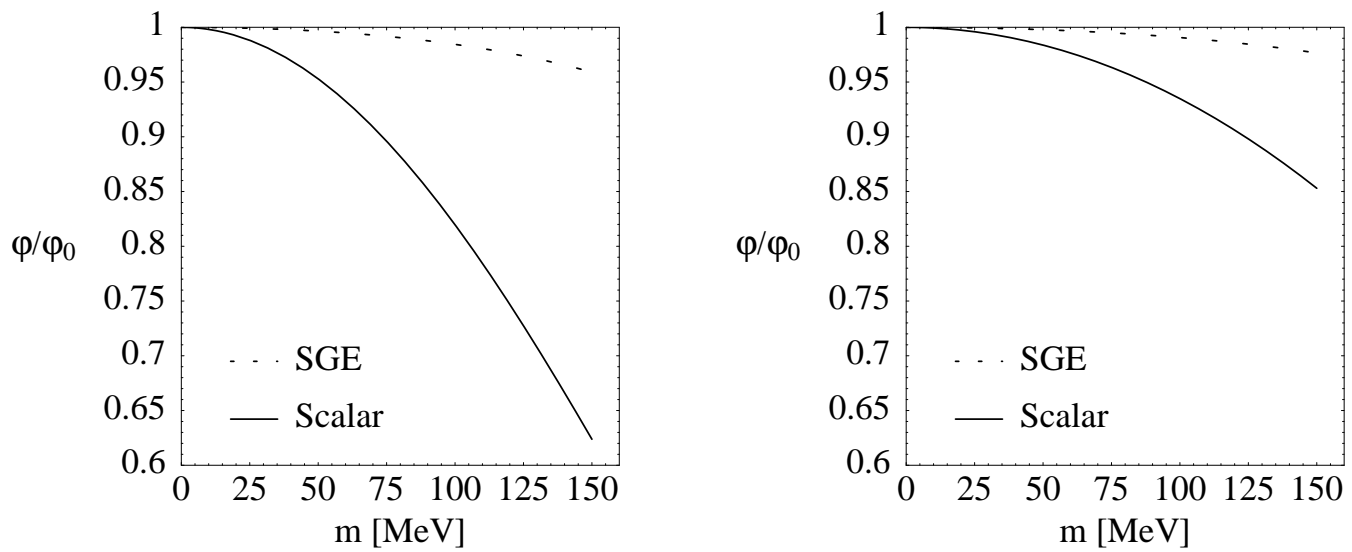

Figure 10: Comparison of the full gap calculations in the scalar NJL model and the single gluon exchange motivated NJL model. The graph on the left is for $G=3.5 \mathrm{GeV}^{-2} / \pi^{2}$ and $\Delta_{1}^{(0)}=10.14 \mathrm{MeV}$. The graph on the right is for $G=6 \mathrm{GeV}^{-2} / \pi^{2}$ and $\Delta_{1}^{(0)}=103.27 \mathrm{MeV}$. 
is no reason to restrict the analysis to interactions of this form. The most general NJL model would involve both types of interactions. This analysis shows that there could be a significant dependence on the specific NJL model used.

\section{Conclusion}

In this paper the general quasiparticle propagator for the case of equally massive quarks is derived. The quasiparticle propagator is then specialized to the propagator in an NJL model. It is shown that there is exactly one new condensate, $\Delta_{3}$, in this model.

The quasiparticle propagator was used to solve the gap equations for the CFL condensate, $\Delta_{1}$, and the new condensate, $\Delta_{3}$ in two NJL models as a function of the quark mass. Approximate analytic solutions were obtained in both cases valid over a range of values of the parameters $m, \Lambda, \mu$ and $G$ in both models. The accuracy of these approximations was tested by numerically solving the exact gap equations. This represents a significant advance as the analytic approximations render numerical solution of the gap equations unnecessary for a significant range of parameter space.

Results for the condensates, $\Delta_{1}$ and $\Delta_{3}$, and the gap, $\varphi$, in the scalar NJL model were presented as a function of the quark mass. The full solution for $\Delta_{1}$ and $\varphi$ were compared to solutions obtained neglecting $\Delta_{3}$. This was done for different values of the four fermion coupling constant. The results show that the complete solution differs from the approximate solution especially at large quark mass and smaller $\Delta_{1}$.

Results for the condensates, $\Delta_{1}$ and $\Delta_{3}$, and the gap, $\varphi$, in the NJL model with the structure of single gluon exchange were presented as a function of the quark mass. The full solution for $\Delta_{1}$ and $\varphi$ were compared to solutions obtained neglecting $\Delta_{3}$. This was again done for different values of the four fermion coupling constant. The results show that the complete solution differs from the approximate solution especially at large quark mass and smaller $\Delta_{1}$.

The solutions obtained in this paper are relevant to the work of [22] where $\Delta_{3}$ was neglected as a first approximation. The approach of this paper could be used to generalize their analysis to the complete case.

The authors of [21] showed that $\Delta_{3}$ could be quite large in an NJL model with a Lorentz non-invariant interaction and have a significant effect on the 
gap. In the analysis presented here it is shown that even for small values of $\Delta_{3}$, neglecting this condensate can have a non-trivial effect on the gap.

Comparison of the results for the gap in the two different NJL models analyzed in this paper show that the gap is strongly dependent on the type of NJL model used.

Solving the color superconducting gap equations for the case of equal mass quarks is also valuable as a precursor to analyzing the physical case where the up and down quarks are essentially massless and the strange quark is massive. The methods used in this research combined with the methods of [15] can be combined to analyze the physical case which is the ultimate goal of this line of research.

\section{Acknowledgments}

I would like to thank D. Rischke, R. Pisarski, T. Schäfer and J. Lenaghan for many valuable discussions and comments. This research was funded by a Natural Science and Engineering Research Council (NSERC) of Canada Post Doctoral Fellowship. This work was also supported in part by DOE grant DE-AC02-98CH10886.

\section{A Nambu-Gorkov Formalism}

In the standard Nambu-Gorkov formalism one deals with the eight component spinors:

$$
\Psi \equiv\left(\begin{array}{c}
\psi \\
\psi_{C}
\end{array}\right), \quad \bar{\Psi} \equiv\left(\begin{array}{ll}
\psi & \psi_{C}
\end{array}\right),
$$

where $C$ is the charge conjugation operator. The action for interacting fermions in the mean field approximation can be written concisely as:

$$
I[\Psi, \bar{\Psi}]=\frac{1}{2} \int \bar{\Psi} \mathcal{S}^{-1} \Psi,
$$

where:

$$
\mathcal{S}^{-1}=\left(\begin{array}{cc}
{\left[G_{0}^{+}\right]^{-1}} & \Delta^{-} \\
\Delta^{+} & {\left[G_{0}^{-}\right]^{-1}}
\end{array}\right) .
$$

$G_{0}^{ \pm}$are the free propagators of particles and charge-conjugate particles, $\Delta^{+}$ is a matrix of diquark condensates and $\Delta^{-} \equiv \gamma_{0} \Delta^{+} \gamma_{0}$. 
The full Nambu-Gorkov propagator is:

$$
\mathcal{S}=\left(\begin{array}{cc}
G^{+} & -G_{0}^{+} \Delta^{-} G^{-} \\
-G_{0}^{-} \Delta^{+} G^{+} & G^{-}
\end{array}\right),
$$

where:

$$
G^{ \pm} \equiv\left\{\left[G_{0}^{ \pm}\right]^{-1}-\Delta^{\mp} G_{0}^{\mp} \Delta^{ \pm}\right\}^{-1}
$$

are the full quasiparticle and charge-conjugate quasiparticle propagators. The gap equation is a consistency equation 27:

$$
\Delta^{+}(k)=-i g^{2} \int \frac{d^{4} q}{(2 \pi)^{4}} \sum_{A, B=1 . . N_{c}^{2}-1} \bar{\Gamma}_{\mu}^{A} D_{A B}^{\mu \nu}(k-q) G_{0}^{-}(q) \Delta^{+}(q) G^{+}(q) \Gamma_{\nu}^{B} .
$$

which must be satisfied in order that the effects of interaction at the mean field level are consistently incorporated in the action (A2) in terms of the diquark condensate matrix $\|^{+}, D_{A B}^{\mu \nu}(k-q)$ is the gluon propagator, $\Gamma_{\nu}^{B}$ is the interaction vertex, and:

$$
\bar{\Gamma}=C \Gamma^{T} C^{-1}
$$

\section{B Dirac Basis}

The Bailin and Love motivated decomposition given in [27] is:

$\Delta=\Delta_{1} \gamma_{5}+\Delta_{2} \gamma \cdot \hat{k} \gamma_{0} \gamma_{5}+\Delta_{3} \gamma_{0} \gamma_{5}+\Delta_{4}+\Delta_{5} \gamma \cdot \hat{k} \gamma_{0}+\Delta_{6} \gamma \cdot \hat{k}+\Delta_{7} \gamma \cdot \hat{k} \gamma_{5}+\Delta_{8} \gamma_{0}$

where:

$$
\Delta_{1} \gamma_{5}+\Delta_{2} \gamma \cdot \hat{k} \gamma_{0} \gamma_{5}
$$

represents condensation of fermions with the same chirality in the even parity channel,

$$
\Delta_{4}+\Delta_{5} \gamma \cdot \hat{k} \gamma_{0}
$$

represents condensation of fermions with the same chirality in the odd parity channel.

$$
\Delta_{3} \gamma_{0} \gamma_{5}+\Delta_{7} \gamma \cdot \hat{k} \gamma_{5}
$$

\footnotetext{
${ }^{4} \Delta^{+}$is also called the gap matrix since it elements determine the gaps in the quasiparticle spectrum.
} 
represents condensation of fermions with opposite chirality in the even parity channel,

$$
\Delta_{6} \gamma \cdot \hat{k}+\Delta_{8} \gamma_{0}
$$

represents condensation of fermions with opposite chirality in the odd parity channel.

\section{References}

[1] B.C. Barrois, Nucl.Phys. B129 (1977) 390.

[2] D. Bailin and A. Love, Phys. Rep. 107 (1984) 325.

[3] M. Alford, K. Rajagopal and F. Wilczek Phys.Lett. B422 (1998) 247 hep-ph/9711395; R. Rapp, T. Schaefer, E. Shuryak and M. Velkovsky, Phys.Rev.Lett. 81 (1998) 53 hep-ph/9711396.

[4] T. Schäfer and E. Shuryak, Lect.Notes Phys. 578 (2001) 203 nuclth/0010049].

[5] K. Rajagopal and F. Wilczek, The Condensed Matter Physics of QCD, Chapter 35 in the Festschrift in honor of B. L. Ioffe, "At the Frontier of Particle Physics / Handbook of QCD", M. Shifman, ed., (World Scientific) hep-ph/0011333.

[6] M. Alford, J. Bowers and K. Rajagopal, J.Phys.G27 (2001) 541 hepph/0009357.

[7] G.W. Carter, Color Superconductivity and Blinking Proto-Neutron Stars, hep-ph/0111353.

[8] M. Prakash, Nucl.Phys. A698 (2002) 440, hep-ph/0105158.

[9] J. Pons, A. Steiner, M. Prakash, J. Lattimer, Phys.Rev.Lett. 86 (2001) 5223, astro-ph/0102015. G.W. Carter and S. Reddy, Phys.Rev. D62 (2000) 103002 [hep-ph/0005228].

[10] M. Alford, K. Rajagopal and F. Wilczek, Nucl.Phys. B537 (1999) 443 hep-ph/9804403. 
[11] T. Schäfer and F. Wilczek, Phys.Rev.Lett. 82 (1999) 3956 hepph/9811473|;

R. Rapp, T. Schäfer, E.V. Shuryak and M. Velkovsky, Annals Phys. 280 (2000) 35 hep-ph/9904353;

T. Schäfer, Nucl.Phys. B575 (2000) 269 hep-ph/9909574;

I. Shovkovy and L. Wijewardhana, Phys.Lett. B470 (1999) 189 hep$\mathrm{ph} / 9910225]$

N. Evans, J. Hormuzdiar, S. Hsu and M. Schwetz, Nucl.Phys. B581 (2000) 391 hep-ph/9910313.

[12] M. Alford, J. Bowers and K. Rajagopal, Phys.Rev. D63 (2001) 074016 hep-ph/0008208; K. Rajagopal, Nucl.Phys. A702 (2002) 25 hep$\mathrm{ph} / 0109135$.

[13] P.F. Bedaque and T. Schäfer, Nucl.Phys. A697 (2002) 802 hepph/0105150;

P.F. Bedaque, Phys.Lett. B524 (2002) 137 [nucl-th/0110049].

[14] D. Kaplan and S. Reddy, Phys.Rev. D65 (2002) 054042 hep$\mathrm{ph} / 0107265$.

[15] T. Fugleberg, Color Superconductivity with Non-Degenerate Quarks, hep-ph/0112162.

[16] M. Alford, J. Berges and K. Rajagopal, Nucl.Phys. B558 (1999) 219 hep-ph/9903502.

[17] T. Schäfer and F. Wilczek, Phys.Rev. D60 (1999) 074014 hepph/9903503.

[18] K. Rajagopal and F. Wilczek Phys.Rev.Lett. 86 (2001) 3492 hep$\mathrm{ph} / 0012039$.

[19] J. Bowers, J. Kundu, K. Rajagopal and E. Shuster, Phys.Rev. D64 (2001) 014024 hep-ph/0101067.

[20] M. Buballa and M. Oertel, Nucl.Phys. A703 (2002) 770 hepph/0109095.

[21] M. Buballa, J. Hosek and M. Oertel, Phys.Rev. D65 (2002) 014018 hepph/0105079. 
[22] M. Huang, P. Zhuang and W. Chao, Phys.Rev. D65 (2002) 076012 hep-ph/0112124.

[23] J. Kundu and K. Rajagopal, Phys.Rev. D65 (2002) 094022 hepph/0112206.

[24] T. Schäfer, Phys.Rev. D65 (2002) 074006 hep-ph/0109052.

[25] A. Leibovich, K. Rajagopal and E. Shuster, Phys.Rev. D64 (2001) 094005 hep-ph/0104073.

[26] M. Harada and S. Takagi, Prog.Theor.Phys. 107 (2002) 561 hepph/0108173.

[27] R. Pisarski and D. Rischke, Phys.Rev. D60 (1999) 094013 nuclth/9903023. 